\title{
Hand Gesture-Based Control of a Front-End Loader
}

\author{
Johann von Tiessenhausen \\ Ingenuity Labs \\ Queen's University \\ Kingston, Canada \\ 13jvt@queensu.ca
}

\author{
Unal Artan \\ Ingenuity Labs \\ Queen's University \\ Kingston, Canada \\ u.artan@queensu.ca
}

\author{
Joshua A. Marshall \\ Ingenuity Labs \\ Queen's University \\ Kingston, Canada \\ ORCID 0000-0002-7736-7981
}

\author{
Qingguo Li \\ Ingenuity Labs \\ Queen's University \\ Kingston, Canada \\ q13@queensu.ca
}

\begin{abstract}
In this paper, we present the design and use of an instrumented glove consisting of a 9-DOF inertial measurement unit (IMU) and resistive flex sensors. The glove is used as a unique human-machine interface to control a Kubota R520s front-end loader, through input gestures, for the excavation of a fragmented rock pile. Raw sensor data from the glove is recorded and transmitted to a computer for gesture recognition. Recognized gestures are then used to command the loader to switch between dig states and control the excavation process. The system allows an operator to observe the entire process from beside the loader, providing them with valuable information about interactions between the loader bucket and rock pile not usually available when seated in the vehicle's cab. Preliminary experiments show that a novice operator was able to improve their performance by using the proposed system, evaluated based on metrics of total and dig completion times, as well as payload.
\end{abstract}

Index Terms-Robotic vehicles, Data glove, Wearable devices

\section{INTRODUCTION}

There is a growing need for robotic vehicle integration in the construction industry due to safety considerations, a projected decrease in qualified work force, and productivity benefits [1], [2]. The integration of robotic machines improves the human operator's safety, reduces their cognitive load through assistance in task completion, and allows them to perform one or multiple tasks with higher efficiency. As such, robotic machines are seen as an invaluable contribution to construction and other heavy industries such as mining, forestry, and agriculture, all of which play a fundamental role in the Canadian economy [3]. Technological advances in other sectors have made the use of robotic vehicles possible, yet there remains a need for efficient collaboration with human operators.

Human operators may be required to perform numerous functions, in collaboration with vehicles of varying autonomy, towards the completion of a task. These functions often require the operator to assume different roles: manual, supervisory, or executive control [4]. Manual control, where the human operator controls all aspects of the robot's operation, is still the most widely used method for controlling construction and other heavy industry machines. Supervisory control requires high-level decision-making to be performed by a human operator, while low-level control is completed by a robotic machine. Executive control affords the robotic machine full autonomy, while the human operator may still be required to start and stop the robot between successive tasks.

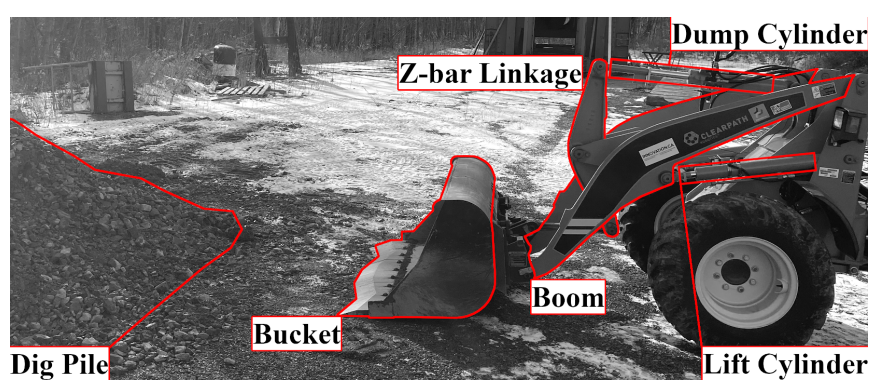

Fig. 1. Operator view of the Kubota R520s front-end loader and rock pile. Lift and dump cylinders shown, with boom and bucket in entry positions.

In this work, we present the design and evaluation of a wearable glove-based gesture recognition system. The system is used to control the excavation of a fragmented rock pile using an instrumented Kubota R520s front-end loader, building on previous works related to fully autonomous excavation [5]. Both discrete and continuous gestural inputs from the operator are used to control the loader. The operator has continuous, manual control of the loader's bucket and throttle during digging. Boom and bucket positions before and after digging are controlled autonomously, while transitions between digging are executed by the operator using discrete inputs.

The operator is able to control the loader from a distance, allowing them to gain valuable information about the interactions between bucket and rock pile. The ability to properly visualize this bucket-pile interaction allows the operator to make decisions that improve dig time and payload acquisition.

To test the feasibility of the proposed system, a single novice operator was asked to perform a simple excavation process on a rock pile by using gesture-based commands. Performance was measured based on the efficiency of the operation, including dig time and payload. The results of preliminary digging experiments are presented, along with a qualitative discussion of the system's behaviour.

\section{RELATED WORK}

\section{A. Autonomous Excavation Background}

In our design and experiments, and based on previous works about autonomous excavation [5], [6], the excavation process consists of the following finite states:

1) Pre-Pile. The boom and bucket are moved to pile entry positions, seen in Fig. 1 


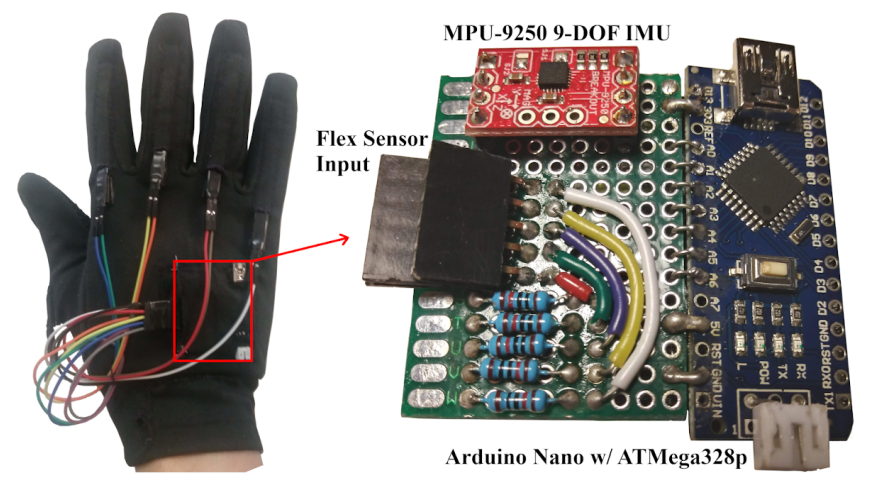

Fig. 2. Proposed prototype. The micro-controller, IMU, and flex sensor inputs are located on the back of the hand, sewn into the glove's liner.

2) Pile. The loader drives forward into the rock pile, maintaining boom and bucket positions.

3) Dig. Once the pile has been penetrated, the dump cylinder is extended to curl the bucket and acquire payload.

4) Post-Dig. Once the dump cylinder has fully extended, the loader is reversed out of the pile and the lift cylinder extended to raise the boom to a post-dig position. The post-dig position is maintained for $10 \mathrm{~s}$ to allow for static load analysis of the final payload.

\section{B. Wearable Glove-Based Devices}

A wearable glove-based device was developed, inspired by existing systems [7]. Such systems for control of mobile robots commonly include an inertial measurement unit (IMU) to measure hand orientation and resistive flex sensors to measure individual finger motion, with the goal of estimating hand pose. Systems using IMUs typically place it on the back of the hand to capture simple dynamic gestures (wave, swipe, trace circle) as discrete inputs [8], [9], or hand pose (roll, pitch) as continuous inputs [10], to control a mobile robot. All sensors are supported using cloth to allow for ease of use across a range of users, however calibration of the flex sensors is required for users of differing hand sizes [11].

\section{PROPOSED SYSTEM}

\section{A. Instrumented Glove}

The glove components, shown in Fig. 2, consist of a micro-controller and a 9-degree-of-freedom IMU sewn into the glove lining on the back of the hand. Inserted into the lining along the length of each finger are resistive flex sensors, connected to the micro-controller. The IMU, an MPU-9250, has 16-bit ADCs that output accelerometer, gyroscope, and magnetometer measurements. Operating ranges of $\pm 4 \mathrm{~g}$ and $\pm 1000^{\circ} / \mathrm{s}$ were selected for the accelerometer and gyroscope, respectively. Analog readings from the flex sensors are converted to 8-bit values by the micro-controller's ADC. IMU and flex sensor outputs are transmitted via serial connection using the Robot Operating System (ROS), Kinetic distribution.

Each user must undergo a simple calibration procedure to account for variations in hand size. The user makes a fist,
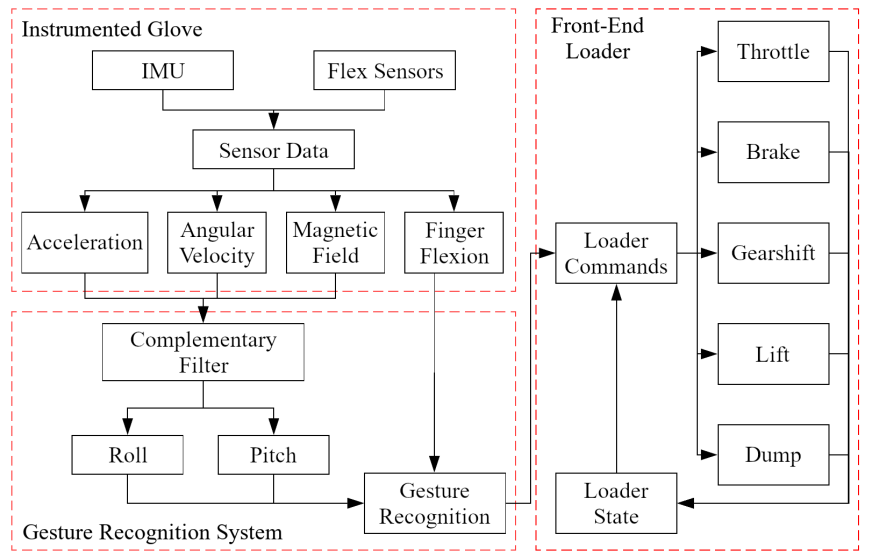

Fig. 3. System architecture. Connections between instrumented glove, gesture recognition system, and front-end loader are shown.

flexing all fingers at once, for approximately $5 \mathrm{~s}$. The user then extends all fingers to form an open palm for an additional 5 s. Measurements from each flex sensor are used to determine relative flexion of each finger. Individual joint flexion angles, therefore, cannot be used for gesture recognition.

\section{B. Gesture Recognition System}

Sensor outputs from the glove are collected and preprocessed by an on-board computer. A complementary filter [12] is used to determine roll and pitch of the user's hand using IMU measurements. Finger flexion is measured as a percentage of maximum flexion. Hand orientation and finger flexion are both used for continuous control of the loader.

Hand pose for discrete gesture classification is determined based on threshold values for hand orientation and finger flexion. Once hand orientation and finger flexion values are within a target range, the discrete gesture is recognized. To determine whether a command may be executed, the system checks whether a discrete gesture has been recognized for a specified period of time. Gestures which move the loader into states that afford control to the operator require higher precision and longer execution times than those which move the loader into idle states, improving safety of operation.

\section{Glove-Loader Interface}

Fig. 3 shows the system architecture of the glove-loader interface. Communication and control of the robotic excavator is achieved via ROS. The master, connected to the loader via an Ethernet connection, receives custom messages containing glove IMU and flex sensor inputs from an ADC at a sampling rate of $125 \mathrm{~Hz}$ via USB connection. Lift and dump cylinder pressures are received from a separate ADC at $125 \mathrm{~Hz}$ via USB connection. Signal preprocessing and gesture recognition are performed in real-time, while state messages are received from the loader at a sampling rate of $10 \mathrm{~Hz}$. State messages provide information regarding lift and dump cylinder positions along with throttle and brake positions, measured using absolute encoders. A callback script is used to publish command outputs, based on loader state messages, at the same rate. 


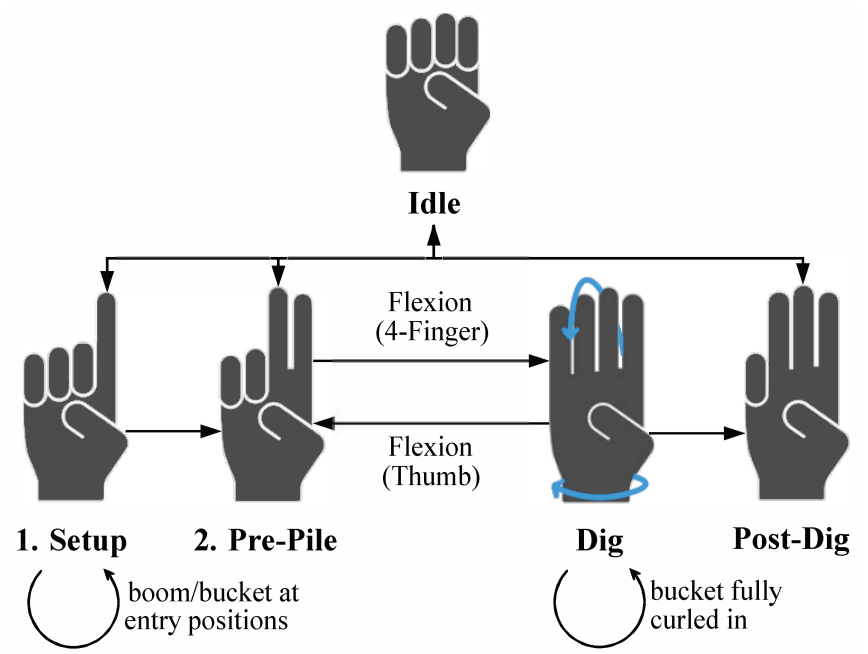

Fig. 4. FSM representation of the loader's control system.

\section{Loader Control}

The Kubota R520s front-end loader, shown in Fig. 1, is equipped with throttle, brake, gearshift, lift, and dump controllers [13]. The loader's operating capacity is specified at $1000 \mathrm{~kg}$, after which the increased strain may lead to an increase in maintenance time and cost. State feedback is provided from position encoders for throttle, brake, lift, and dump. The operator is positioned, as shown, to give a clear view of the bucket-pile interaction throughout the excavation process.

A Finite State Machine (FSM) representation of the loader's control states are shown in Fig. 4. Digging states are selected by holding a Number 1, 2, or 3 gesture for $0.3 \mathrm{~s}$. The Idle state is selected by holding a Closed Fist gesture for $0.1 \mathrm{~s}$. Each gesture corresponds to a loader control state which may be used during, before, or after the excavation process. Loader states may be selected while in the Idle state, and transitions between states occur once certain conditions are met.

Control during the excavation process is described below, building upon on previous works within our lab regarding autonomous excavation [5], [6]:

1) Setup. The operator first starts in the Idle state. They then move to the Setup state by performing a Number 1 gesture. Using closed-loop proportional control with lift and dump position feedback, the lift and dump cylinders are moved to within $5 \mathrm{~mm}$ of their dig positions. Once complete, the loader moves into the Pre-Pile state.

2) Pre-Pile/Dig. Control during the Pre-Pile/Dig states is shown in Fig. 5. To move into the Dig state, the average flexion of the operator's fingers, excluding the thumb, is used (4-finger flexion). Average 4-finger flexion is used to control loader throttle input, and roll of the user's hand is used to control bucket curl through dump cylinder extension. Flexion of the thumb exits the Dig state and prevents both throttle and dump commands. Once the bucket has fully curled in, digging is complete

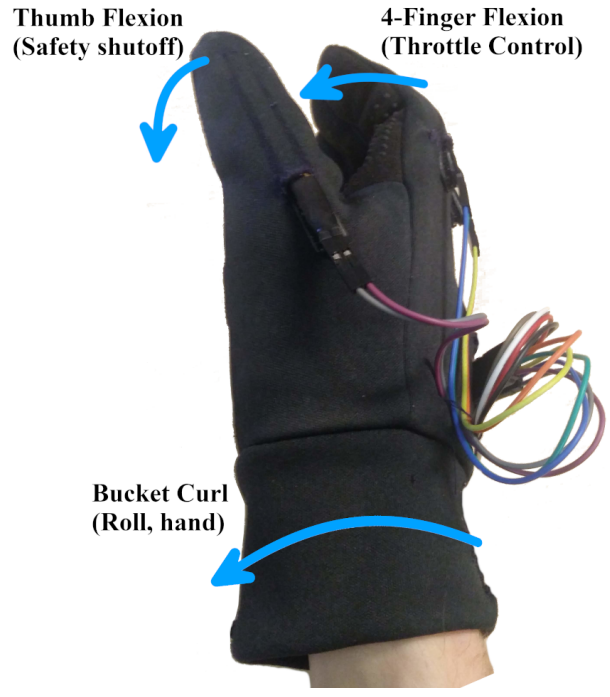

Fig. 5. Glove control diagram for the Pre-Pile/Dig state.

and the loader moves into the Post-Dig state.

3) Post-Dig. The lift cylinder moves the boom to the PostDig position and the operator reverses by using 4 -finger flexion. Once complete, the operator moves back into the Idle state using a Closed Fist gesture.

For larger payloads, a proportional increase in dump command is needed to curl the bucket such that

$$
P=F v,
$$

where $P$ is dump cylinder power in $\mathrm{J} / \mathrm{s}, F$ is payload weight in $\mathrm{N}$, and $v$ is bucket curl velocity in $\mathrm{m} / \mathrm{s}$. The operator is responsible for providing the necessary amount of power through input commands to achieve a desired velocity. Using visual feedback of the bucket-pile interaction, the operator is able to obtain information in real time regarding the approximate payload level and loader throttle. This presents a challenge to the operator, however, because they are required to adjust dump and throttle command inputs simultaneously and with respect to one another.

\section{RESUlTS AND DisCUSSION}

A preliminary assessment of the proposed system was conducted, wherein a single novice operator performed the excavation process 14 consecutive times using the control method described above. Testing occurred in sub-zero temperatures $\left(-16^{\circ} \mathrm{C}\right.$ approximate air temperature), changing the characteristics of the rock pile and making payload acquisition more difficult. The system was evaluated based on completion time of the excavation process from Setup to Post-Dig states, Dig state time, and final payload size.

Completion time gives an indirect measure of a single novice operator's performance using the proposed system. As the operator becomes more familiar with the system, they are able to move between states and move the loader to and from the pile faster. Completion time decreased with subsequent trials $\left(R^{2}=0.652\right)$ as the operator became experienced with 


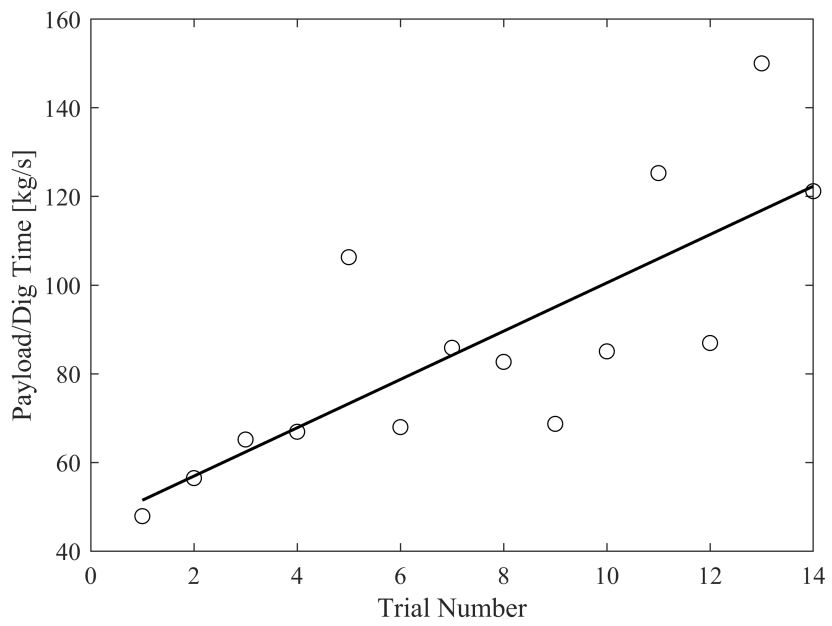

Fig. 6. Results of operator performance during Dig state, measured as a ratio between Payload Size and Dig Time $(\mathrm{kg} / \mathrm{s})$

the excavation process. No relationship between trial number and payload size was found. An average payload size of $741 \pm 111 \mathrm{~kg}$ was achieved across all trials compared to a maximum optimal payload size of $1000 \mathrm{~kg}$, likely due to frozen pile fragments present during experimentation, affecting payload acquisition.

Based on the relationship described in (1), an increased digging force requires a proportional increase in dump power to maintain bucket curl velocity. To maximize final payload and minimize digging time, the operator must adjust dump command inputs based on the bucket-pile interaction. To measure the performance of the operator during the Dig state, a ratio of payload to dig time is used to describe the amount of material the operator is able to acquire during the dig state. The results of this ratio across all trials is shown in Fig. 6. An increase in material acquired over time was found across subsequent trials $\left(R^{2}=0.612\right)$, demonstrating an increase in operator performance as they became more experienced with the digging operation.

\section{CONCLUSION}

The proposed system demonstrates gesture-based control of an instrumented front-end loader and allows for remote control of the system. The operator is able to move freely beside the loader, providing them visual feedback during the excavation process and affording them the ability to perform multiple tasks without needing to remain within the loader's cab.

Based on the results, it was found that a single novice operator was able to properly perform the excavation process using the proposed system, with improvements in performance observed for successive trials. Improvements need to be made, however, to maximize payload acquisition and increase the efficiency of the task.

\section{FUTURE WORK}

Implementing a velocity controller that converts command inputs to bucket velocity outputs could improve the operator's performance during the Dig state, but the use of an integrator to determine output velocity would introduce its own delay. Additional assistance could be provided via a simple buzzer fixed to the back of the operator's hand, providing them with force feedback of the bucket-pile interaction [14]. The use of force feedback in combination with visual feedback of the bucket-pile interaction would help a novice operator determine when to initiate bucket curl during the Dig state.

The current prototype communicates via USB cable, connecting the operator directly to the loader. This poses both a constraint to the operator and a potential safety issue. In the future, wireless communication will be implemented.

\section{REFERENCES}

[1] P. M. Goodrum and C. T. Haas, "Long-term impact of equipment technology on labor productivity in the U.S. construction industry at the activity level," J. Constr. Eng. Manag., vol. 130, no. 1, pp. 124-133, February 2004.

[2] A. M. Lytle and K. S. Saidi, "NIST research in autonomous construction," Auton. Robots, vol. 22, no. 3, pp. 211-221, April 2007.

[3] Statistics Canada, "Gross domestic product (GDP) at basic prices, by industry, annual average," 2018.

[4] D. J. Folds and D. A. Mitta, "Using operator role theory to guide function allocation in system development," Proc. Hum. Factors Ergon. Soc. Аnпu. Meet., vol. 39, no. 18, pp. 1155-1159, October 1995.

[5] H. A. Fernando, J. A. Marshall, H. Almqvist, and J. Larsson, "Towards controlling bucket fill factor in robotic excavation by learning admittance control setpoints," in F. Serv. Robot., 2018, pp. 35-48.

[6] H. Fernando, J. A. Marshall, and J. Larsson, "Iterative learning-based admittance control for autonomous excavation," J. Intell. Robot. Syst. Theory Appl., vol. 96, no. 3-4, December 2019.

[7] L. Dipietro, A. M. Sabatini, S. Member, and P. Dario, "A survey of glove-based systems and their applications," IEEE Trans. Syst. Man Cybern. Part C Appl. Rev., vol. 38, no. 4, p. 461, 2008.

[8] X. H. Wu, M. C. Su, and P. C. Wang, "A hand-gesture-based control interface for a car-robot," in Int. Conf. Intell. Robot. Syst., 2010, pp. 4644-4648.

[9] C. Zhu and W. Sheng, "Wearable sensor-based hand gesture and daily activity recognition for robot-assisted living," IEEE Trans. Syst. Man, Cybern. Part A: Systems Humans, vol. 41, no. 3, pp. 569-573, 2011.

[10] S. O. Shin, D. Kim, and Y. H. Seo, "Controlling mobile robot using imu and emg sensor-based gesture recognition," in Int. Conf. Broadband Wirel. Comput. Commun. Appl. Institute of Electrical and Electronics Engineers Inc., jan 2014, pp. 554-557.

[11] G. D. Kessler, L. F. Hodges, and N. Walker, "Evaluation of the cyberglove as a whole-hand input device," ACM Trans. Comput. Interact., vol. 2, no. 4, pp. 263-283, January 1995.

[12] R. Mahony, T. Hamel, J.-M. Pflimlin, J.-M. Pflimlin Nonlinear, and R. Mahony, "Complementary filters on the special orthogonal group," IEEE Trans. Autom. Control. Inst. Electr. Electron. Eng., vol. 53, no. 5, pp. 1203-1217, 2008.

[13] A. A. Dobson, J. A. Marshall, and J. Larsson, "Admittance control for robotic loading: Design and experiments with a 1-tonne loader and a 14-tonne load-haul-dump machine," J. F. Robot., vol. 34, no. 1, pp. 123-150, January 2017.

[14] M. Dupont, "Reconnaissance gestuelle par gant de données pour le contrôle temps réel d'un robot mobile," Ph.D. dissertation, Université de Bretagne Sud, 2017. 